\title{
Effects of different drying methods on drying kinetics and physicochemical properties of Chrysanthemum morifolium Ramat
}

\author{
Borui Li, Jueyi Lin, Zhian Zheng, Hao Duan, Dong Li, Min Wu* \\ (College of Engineering, China Agricultural University, Beijing 100083, China)
}

\begin{abstract}
The effects of infrared-assisted hot-air drying (IR-HAD), temperature, humidity controlled hot-air drying (THC-HAD), and hot-air drying (HAD) on the drying kinetics, physicochemical properties, chlorogenic acid content and microstructure of chrysanthemum were experimentally examined. The results showed that the drying time reduced with increasing air drying temperature, with IR-HAD needing the shortest drying time, followed by THC-HAD and HAD. The effective moisture diffusivities $\left(D_{e f f}\right)$ of chrysanthemum under IR-HAD, THC-HAD, and HAD at $60^{\circ} \mathrm{C}$ were $3.22 \times 10^{-9} \mathrm{~m}^{2} / \mathrm{s}$, $2.19 \times 10^{-9} \mathrm{~m}^{2} / \mathrm{s}$, and $2.89 \times 10^{-9} \mathrm{~m}^{2} / \mathrm{s}$, respectively. IR-HAD preserved chrysanthemum surface color better than THC-HAD and HAD, whereas the THC-HAD samples obtained higher water holding capacity $(W H C)$, water binding capacity (WBC), and chlorogenic acid content. Additionally, peroxidase (POD) residual activity of the samples decreased with increasing blanching time. The current work provides a theoretical basis for the drying of chrysanthemum.
\end{abstract}

Keywords: chrysanthemum, drying kinetics, Weibull model, physicochemical properties, peroxidase $(P O D)$ residual activity, chlorogenic acid content

DOI: $10.25165 /$ j.ijabe. 20191203.4820

Citation: Li B R, Lin J Y, Zheng Z A, Duan H, Li D, Wu M. Effects of different drying methods on drying kinetics and physicochemical properties of Chrysanthemum morifolium Ramat. Int J Agric \& Biol Eng, 2019; 12(3): 187-193.

\section{Introduction}

Chrysanthemum (Chrysanthemum morifolium Ramat.) is widely planted in Asian countries, and its flower heads have been used as a traditional Chinese herbal medicine ${ }^{[1]}$. Chrysanthemums play a key role in modern flower markets and have both decorative function and economic value ${ }^{[2]}$. Medicinal chrysanthemum can dissipate heat, calm eyesight, reduce headache, vertigo, and to detoxify in our blood and digestive system ${ }^{[3]}$. This herb is a nutritionally potent source of nutrients, such as vitamin A, vitamin $\mathrm{B}_{1}$, vitamin $\mathrm{C}$, and various amino acids ${ }^{[4]}$. Medicinal chrysanthemum contains many flavonoids and polyphenols, with chlorogenic acid being one of the main polyphenols, and it has strong antioxidant activity, inhibits lipid peroxidation, and has anticancer effects ${ }^{[5]}$. In modern times, chrysanthemums are also used as a functional food.

Fresh chrysanthemum flowers are hard to save, and the flavor, color, and nutrients quickly deteriorate. Fresh flowers have a high moisture content, which can reach more than $80 \%$. Drying is an important step after chrysanthemum harvest, because it can limit enzyme degradation and microbial growth while retaining the beneficial characteristics of the flowers ${ }^{[6]}$. Chrysanthemum

Received date: 2018-12-04 Accepted date: 2019-04-21

Biographies: Borui Li, Master candidate, research interests: food science and engineering, Email: boruili@cau.edu.cn; Jueyi Lin, Bachelor, research interests: agricultural engineering, Email:1160868336@qq.com; Zhian Zheng, PhD, Associate Professor, research interests: agricultural engineering, Email: zhengza@cau.edu.cn; Hao Duan, Master candidate, research interests: drying technology and engineering, Email: 13891037020@163.com; Dong Li, PhD, Professor, research interests: agricultural engineering, Email: dongli@ cau.edu.cn.

*Corresponding author: Min Wu, PhD, Assistant Professor, research interests: agricultural engineering, drying technology and engineering, College of Engineering, China Agricultural University; Engineering Research Center for Modern Agricultural Equipment \& Facilities. P. O. Box 50, 17 Qinghua Donglu, Beijing 100083, China. Tel/Fax: +86-1062736883; E-mail: minwu@ cau.edu.cn. flowers contain many enzymes, such as polyphenol oxidase (PPO), peroxidase (POD) and superoxide dismutase $(\mathrm{SOD})^{[7]}$, which can adversely affect quality, and so an enzyme-killing pretreatment is usually performed before drying. High-humidity hot air impingement blanching (HHAIB), based on high temperature and high-humidity gas, comprehensively utilizes the characteristics of high convective heat transfer coefficient and enthalpy to inactivate flower enzymes ${ }^{[8]}$, and also improves the drying rate ${ }^{[9]}$. In recent years, HHAIB has been applied to a range of food and agricultural products, including to dry seedless grapes ${ }^{[10]}$, apricots ${ }^{[11]}$, and American ginseng slices ${ }^{[12]}$.

In China, the traditional chrysanthemum flower drying method is open-air sun drying, but this takes too long a time. At present, hot air drying is the most universal drying method for agricultural products. Unfortunately, this type of drying reduces product quality by affecting chemical properties and reducing principal active ingredients ${ }^{[13]}$. Color, surface morphology, and active ingredients of different products were destroyed by the hot air-drying process ${ }^{[13,14]}$.

Infrared drying has a high drying rate, and in brown rice, showed positive effects in terms of the color, microstructure, cooking, gelatinized texture, and pasting characteristics ${ }^{[15]}$. Wang et al. ${ }^{[1]}$ found that combined hot air and microwave-assisted drying can significantly increase the effective moisture diffusivities $\left(D_{\text {eff }}\right)$ and more chemical ingredients and antioxidant activities could be extracted from chrysanthemum flowers. Activation energy $(E a)$ increased with increasing air flow velocity and decreasing IR radiation intensity ${ }^{[16]}$. However, the effect of relative humidity $(R H)$ on drying characteristics and product quality has mainly focused on fruits and vegetables, and there is no research on the effect of RH on chrysanthemum drying.

When chrysanthemum flowers were dried using open-air sun drying, shade drying, or oven drying, drying time in the oven was shortest, but reduced the active ingredient content ${ }^{[6]}$. Wang et al. ${ }^{[1]}$ focused on the effect of hot air and microwave-assisted drying on 
the energy consumption of chrysanthemum, but did not analyze physicochemical properties. However, another study reported that drying temperature, relative air humidity, sample thickness, and different step-down relative humidity are all the factors that affect the drying kinetics of chrysanthemum ${ }^{[17]}$.

This research explored the effects of IR-HAD, THC-HAD, and HAD on the drying kinetics, physicochemical properties, and microstructure of chrysanthemum flowers under various drying temperatures $\left(50^{\circ} \mathrm{C}, 60^{\circ} \mathrm{C}, 70^{\circ} \mathrm{C}\right.$, and $\left.80^{\circ} \mathrm{C}\right)$. This research addresses the need to select a suitable drying technology for medical chrysanthemum.

\section{Materials and methods}

\subsection{Materials}

Chrysanthemums (Chrysanthemum morifolium Ramat.) were provided from $\mathrm{HuBei}$, China and stored in a refrigerator at $4^{\circ} \mathrm{C} \pm 1^{\circ} \mathrm{C}$. Mechanically damaged and decayed chrysanthemums were removed, and chrysanthemums with a uniform color and size were manually selected as the blanching samples. Before the experiments, the samples were cleaned to remove any dust on their surface. The average initial moisture content of the chrysanthemum samples was $83.34 \% \pm 0.17 \%$ (wet basis, w.b.), with the moisture determined according to the National Standard GB/T 5009.3-2016 ${ }^{[18]}$.

\subsection{HHAIB pretreatment of the chrysanthemum samples}

HHAIB equipment was installed at the College of Engineering of China Agricultural University, Beijing, China. The equipment has previously been described in detail by Bai et al. ${ }^{[19]}$. A schematic diagram of the equipment is shown in Figure 1. According to previous studies ${ }^{[20]}$ and a preliminary experiment, an air velocity of $14.0 \pm 0.5 \mathrm{~m} / \mathrm{s}$, a temperature of $95^{\circ} \mathrm{C}$, and a hot air relative humidity of $40 \%-45 \%$ were used as the optimal blanching conditions. The sample was placed flat over stainless steel wire of the impingement blanching machine. Then they were dealt with $H H A I B$ for $20 \mathrm{~s}, 40 \mathrm{~s}, 50 \mathrm{~s}$, or $60 \mathrm{~s}$. The blanched chrysanthemums were cooled to room temperature and then dried.

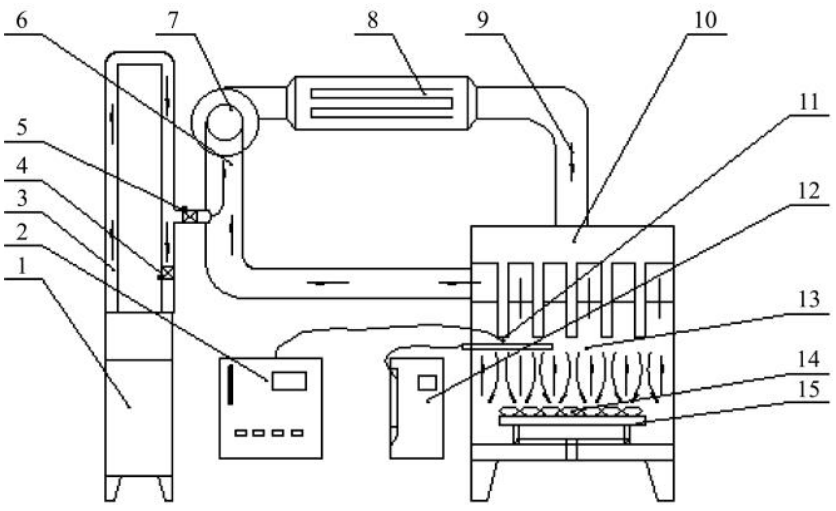

Figure 1 Schematic diagram of equipment used for high humidity air impingement blanching (HHAIB) treatment

\subsection{POD activity}

POD activity was determined according to the kit method (A084-3, Nanjing Jiancheng, China). Chrysanthemum samples were weighed to $0.001 \mathrm{~g}$ accuracy, and then blended using a mortar and pestle in a phosphate buffer $(0.1 \mathrm{~mol} / \mathrm{L}, \mathrm{pH} 7.0)$ with a ratio of $1: 9$ (w:v), to obtain a $10 \%$ tissue homogenate under an ice water bath. The homogenate was centrifuged in a high-speed centrifuge (GL-20G-II, Anke Instrument Co. Ltd., Shanghai, China) at $11180 \times g$ for $10 \mathrm{~min}$, at a temperature of $4^{\circ} \mathrm{C}$. The POD activity in the supernatant was determined using a spectrophotometer (TU-1810, Beijing Purkinje General Instrument Co. Ltd., Beijing, China) to measure the increased absorbance at $420 \mathrm{~nm}$. A change in the absorbance value of 0.001 was defined to be one unit of enzyme activity. The results are expressed as the relative activity (\%) compared to the initial value.

\subsection{Drying methods}

In this study, three kinds of drying techniques were used to dry chrysanthemum flowers, which were hot air-drying, temperature and humidity controlled hot air-drying, and infrared radiation-assisted hot air-drying. The samples (weighing about $300 \mathrm{~g}, 300 \mathrm{~g}$, and $200 \mathrm{~g}$, for the HAD, IR-HAD, and THC-HAD samples, respectively) were placed flat over a stainless steel wire, then put the wire into drying chamber. All of the drying tests were carried out in triplicate.

\subsubsection{Hot air-drying}

The HAD equipment was as previously described by Ju et al. ${ }^{[17]}$ The drying temperatures were performed at $50^{\circ} \mathrm{C}, 60^{\circ} \mathrm{C}, 70^{\circ} \mathrm{C}$, and $80^{\circ} \mathrm{C}$, respectively ${ }^{[21]}$. The wind velocity of hot air drying was $1.5 \mathrm{~m} / \mathrm{s}$, and the direction was parallel to the drying tray.

2.4.2 Infrared radiation-assisted hot air-drying

The IR-HAD equipment was equipped with three $450 \mathrm{~W}$ infrared lamps on the basis of the dryer (STC Taizhou Senttech Infrared Technology Co., Ltd, Jiangsu, China). The drying temperatures were set to $50^{\circ} \mathrm{C}, 60^{\circ} \mathrm{C}, 70^{\circ} \mathrm{C}$, and $80^{\circ} \mathrm{C}$, respectively. Hot air flow velocity was $3.0 \mathrm{~m} / \mathrm{s}$, and the direction was parallel to the drying tray.

\subsubsection{Temperature and humidity controlled hot air-drying}

The experiments were carried out using a laboratory scale hot air dryer with humidity control as described in detail ${ }^{[17]}$. According to the preliminary experiment, the experiments were performed under stepped-down relative humidity conditions. The relative humidity was $50 \%$ for $90 \mathrm{~min}$ to a $20 \% \mathrm{RH}$ in the end, at $50^{\circ} \mathrm{C}, 60^{\circ} \mathrm{C}, 70^{\circ} \mathrm{C}$, and $80^{\circ} \mathrm{C}$ drying temperature, and with a constant air velocity of $1.5 \mathrm{~m} / \mathrm{s}$.

\subsection{Analysis of the drying characteristics}

The weight loss of the samples was calculated by removing the drying tray from the chamber and weighing it using an electronic balance (SP402, Ohaus Co., New Jersey, USA). The sample weight was recorded every $10 \mathrm{~min}$ in the first hour and every 30 min thereafter, until the products reached the desired moisture content ( $<10 \%$ w.b.), which is considered a safe level for long-term storage of chrysanthemum ${ }^{[22]}$. It took less than $10 \mathrm{~s}$ to weigh each sample. The average moisture contents were determined and used to draw the drying curves. After drying, the samples were immediately stored in a $4^{\circ} \mathrm{C}$ refrigerator.

\subsubsection{Moisture ratio}

The moisture ratios $(M R)$ of the chrysanthemums were calculated using the following equation Equation (1):

$$
M R=\frac{M_{t}}{M_{0}}
$$

where, $M_{t}$ is the moisture content at a drying time $t, \mathrm{~kg} / \mathrm{kg}$ (dry basis, d.b.); and $M_{0}$ is the initial moisture content.

The drying rate $(D R)$ of the chrysanthemum at a particular time period was calculated by Equation (2):

$$
D R=\frac{M_{t_{1}}-M_{t_{2}}}{t_{2}-t_{1}}
$$

where, $t_{1}$ and $t_{2}$ are the drying times, h; and $M_{t_{1}}$ and $M_{t_{2}}$ are the moisture contents, $\mathrm{kg} / \mathrm{kg}$ (dry basis, d.b.) at times $t_{1}$ and $t_{2}$, respectively. 


\subsubsection{Weibull model}

The Weibull model is extensively applied to describe the moisture changes of materials under different drying conditions ${ }^{[23]}$. The Weibull distribution function was calculated according to Equation (3):

$$
M R=\exp \left[-\left(\frac{t}{\alpha}\right)^{\beta}\right]
$$

where, $\alpha$ is the scale parameter of the Weibull model; $\beta$ is the shape parameter of the model, and $t$ is the drying time in minutes.

\subsubsection{Effective moisture diffusivity $\left(D_{e f f}\right)$}

The law for moisture diffusion in the drying process can be described by Fick's second law. The relationship between the moisture ratio $(M R)$ and $D_{\text {eff }}$ can be expressed as Equation (4):

$$
M R=\frac{8}{\pi^{2}} \sum_{n=1}^{\infty} \frac{1}{n^{2}} \exp \left(-\frac{\pi^{2} D_{\text {eff }}}{H^{2}} t\right)
$$

For long drying times, Equation (4) can be further simplified to the straight-line equation of Equation (5):

$$
M R=\frac{8}{\pi^{2}} \exp \left(-\frac{\pi^{2} D_{e f f}}{H^{2}} t\right)
$$

where, $D_{\text {eff }}$ is the effective moisture diffusivity, $\mathrm{m}^{2} / \mathrm{s} ; H$ is the thickness of the chrysanthemum (with a value of $7.2 \times 10^{-3} \mathrm{~m}$ in this study); and $t$ is the drying time, s.

Taking the natural logarithm of both sides of Equation (5), we obtain Equation (6):

$$
\ln M R=\ln \frac{8}{\pi^{2}}-\frac{\pi^{2} D_{e f f}}{H^{2}} t
$$

Therefore, the slope $(k)$ can be obtained from the linear regression of $\ln (M R)$ versus the time curves, and the $D_{\text {eff }}$ can be obtained by Equation (7):

$$
D_{e f f}=-\frac{H^{2}}{\pi^{2}} k
$$

\subsection{Color measurement}

The color of chrysanthemum flowers was measured before and after drying using a D65 colorimeter (WR-10, Wei Fu Guang Dian Co. Ltd., Shenzhen, China) as described ${ }^{[24]}$. Color was expressed in CIELAB, i.e., $L^{*}$ (lightness), $a^{*}$ (redness or greenness), and $b^{*}$ (yellowness or blueness) coordinates, with a standard D65 illuminant and a measuring caliper of $8 \mathrm{~mm}$. The total color changes were calculated by Equation (8):

$$
\Delta E=\sqrt{\left(L^{*}-L_{0}^{*}\right)^{2}+\left(a^{*}-a_{0}^{*}\right)^{2}+\left(b^{*}-b_{0}^{*}\right)^{2}}
$$

where, $\Delta E$ is the difference between the color of the fresh and dried chrysanthemum samples; $L_{0}{ }^{*}, a_{0}{ }^{*}$, and $b_{0}{ }^{*}$ are the color parameters of the freeze-dried chrysanthemum; and $L^{*}, a^{*}$, and $b^{*}$ are the color parameters of the dried samples.

\subsection{Determination of chlorogenic acid content}

Chlorogenic acid content was measured based on the Chinese pharmacopoeia $(2015)^{[3]}$. Each sample was analyzed using high performance liquid chromatography (HPLC) on an UltiMate3000 (DIONEX, America). A C18 rapid resolution analysis column (5 $\mu \mathrm{m}, 250 \mathrm{~mm} \times 4.6 \mathrm{~mm}$, Shimadzu, Japan) was used, with a dual solvent mobile phase of solvents A (acetonitrile) and B (0.1\% phosphoric acid, v/v). The program for the gradient elution was: $10 \%-18 \% \mathrm{~A}$ and $90 \%-82 \% \mathrm{~B}$ at $0-11 \mathrm{~min}, 18 \%-20 \% \mathrm{~A}$ and $82 \%-80 \% \mathrm{~B}$ at $11-30 \mathrm{~min}, 20 \% \mathrm{~A}$ and $80 \% \mathrm{~B}$ at $30-55 \mathrm{~min}$. The flow rate was $1.0 \mathrm{~mL} / \mathrm{min}$ and the injection volume was $5 \mu \mathrm{L}$. The detection wavelength was $348 \mathrm{~nm}$ with a column temperature at $30^{\circ} \mathrm{C}$.
Preparation of the sample solution: about $0.25 \mathrm{~g}$ of chrysanthemum powder was weighed to $0.001 \mathrm{~g}$ accuracy, put into a $50 \mathrm{~mL}$ conical flask. $25 \mathrm{~mL}$ of $70 \%$ methanol was added, followed by ultrasonic treatment $(40 \mathrm{kHz} 200 \mathrm{~W}, 40 \mathrm{~min})$, return to room temperature and weighed again. $70 \%$ methanol was used to complement the reduced weight, and then the sample was shaken and filtered, and the filtrate was obtained.

Preparation of the reference solution: a proper amount of chlorogenic acid reference substance was added in a $25 \mathrm{~mL}$ brown bottle, and $70 \%$ methanol was added to produce a solution containing chlorogenic acid $(45.0,22.5,11.25$, and $5.62 \mu \mathrm{g} / \mathrm{mL}$ ), which was stored below $10^{\circ} \mathrm{C}$.

Linear relation investigation: the chlorogenic acid reference solution was precisely taken and injected into the HPLC. The peak area was determined according to the aforementioned chromatographic conditions. With the peak area as the ordinate and the concentration of chlorogenic acid as the coordinate, the standard curve was drawn and the regression equation was obtained.

\subsection{Determination of the water holding capacity and water binding capacity}

The AACC (2010) method was used to determine the water holding capacity and water binding capacity. Some adjustments were made according to the actual situation of the chrysanthemum samples.

\subsubsection{Determination of water holding capacity}

Dried chrysanthemum powder $(0.5000 \pm 0.0005 \mathrm{~g})$ and deionized water $(25 \mathrm{~mL})$ were thoroughly mixed and placed in a $50 \mathrm{~mL}$ test tube. The mixture was held at room temperature $25^{\circ} \mathrm{C}$ for $24 \mathrm{~h}$, then the liquid was slowly decanted. The water holding capacity of the samples were calculated by Equation (9):

$$
W H C=\frac{m_{0}-m_{d}}{m_{d}}
$$

where, $W H C$ is water holding capacity, $\mathrm{g} / \mathrm{g} ; m_{d}$ is the weight of the raw chrysanthemum powder, and $m_{0}$ is the weight of the chrysanthemum powder after water absorption.

2.8.2 Determination of water binding capacity

Dried chrysanthemum powder $(0.5000 \pm 0.0005 \mathrm{~g})$ and deionized water $(25 \mathrm{~mL})$ were thoroughly mixed and placed in a $50 \mathrm{~mL}$ test tube, then centrifuged for ten minutes in a high-speed centrifuge at a temperature of $4{ }^{\circ} \mathrm{C}$, and with a rotational speed of $11180 \times g$. After the sample was removed, the upper liquid was slowly poured out. Equation (10) was used to calculate the water binding capacity of the samples:

$$
W B C=\frac{m_{0}-m_{d}}{m_{d}}
$$

where, $W B C$ is the water binding capacity, $\mathrm{g} / \mathrm{g} ; m_{d}$ is the weight of the raw chrysanthemum powder, and $m_{0}$ is the weight of the chrysanthemum powder after water absorption.

\subsection{Microstructure}

Dried samples were smashed into powder with a high-speed disintegrator, and were then mounted with a conductive adhesive tape on aluminum stubs and visualized using a scanning electron microscope (S-3400N, Hitachi, Japan). The sample patterns were observed at an accelerating voltage of $15 \mathrm{kV}$. The cells located on the edges of the slices were disregarded in the analysis.

\subsection{Statistical analysis}

The values were expressed as the means \pm standard deviations (SD), and then analyzed by ANOVA and Duncan's multiple-range test using SPSS statistics software (version 21.0, SPSS Inc., 
Chicago, IL, USA) to analyze variance and significant differences. The statistical significance for the differences was tested at a 5\% probability level $(p<0.05)$.

\section{Results and discussion}

\subsection{Effect of HHAIB time on $P O D$ residual activity}

POD is an oxidoreductase naturally present in chrysanthemums, and can be used as an index of the effectiveness of blanching (MV, MR, SI \& CEdel, 2008). The changes in POD activity in blanched chrysanthemum samples under different $H H A I B$ time conditions is observed in Figure 2. Data are expressed as relative activity (\%) relative to fresh chrysanthemum samples. As blanching time increased, the POD activity reduced and dropping to less than $10 \%$ exceeds $40 \mathrm{~s}$.

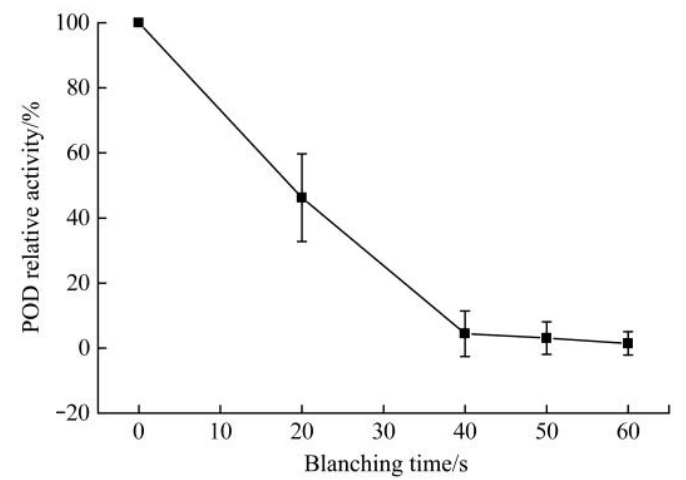

Figure 2 Effect of different HHAIB times (20 s, $40 \mathrm{~s}, 50 \mathrm{~s}$, and $60 \mathrm{~s})$ on POD relative activity in chrysanthemum samples blanched at $95^{\circ} \mathrm{C}$
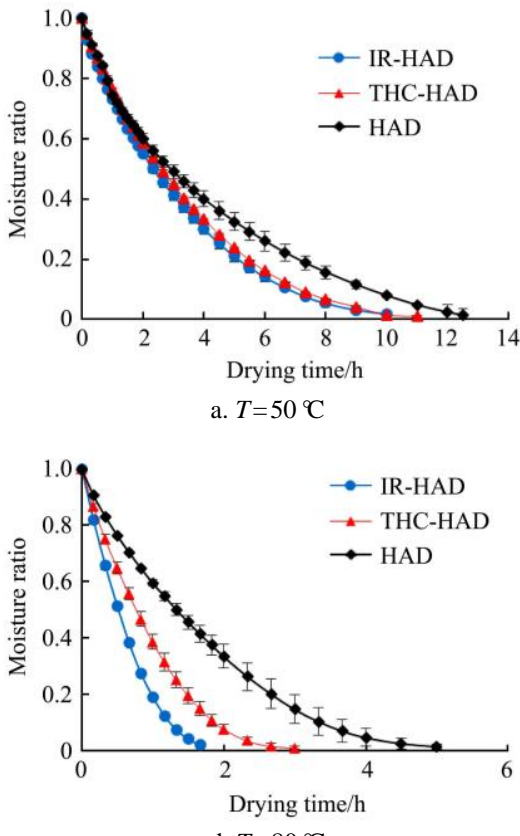

d. $T=80^{\circ} \mathrm{C}$
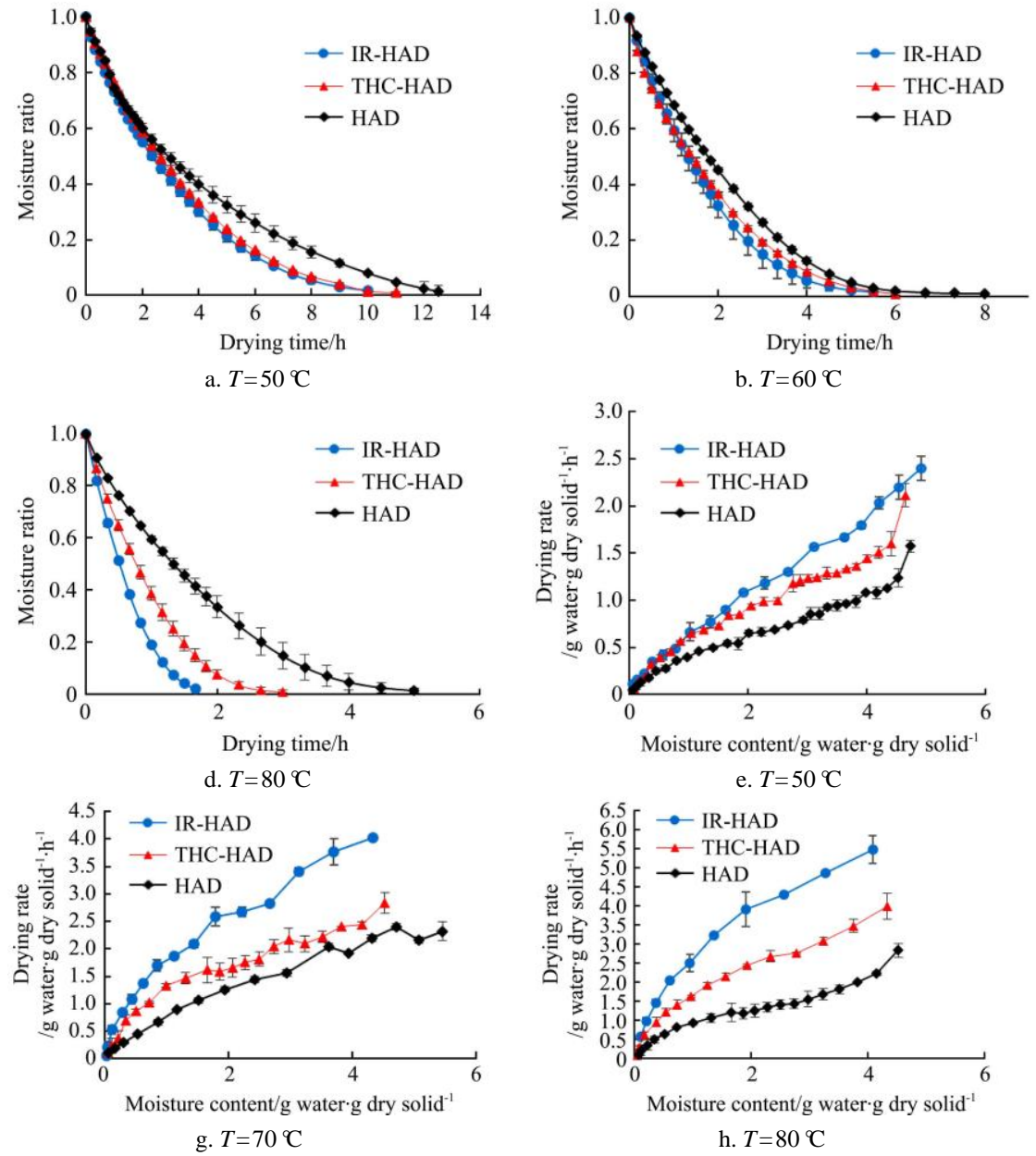

\subsection{Drying characteristics}

\subsubsection{Drying kinetics curves}

All drying methods were able to dry the chrysanthemum samples from $83.34 \%$ (w.b.) to $10 \%$ (w.b.) (Figure 3). The drying time required by the three drying methods shortened as the drying temperature increased, and temperature is the key factor in drying kinetics. A high temperature not only produces a large thermal driving force, but also increases the effective moisture diffusion ${ }^{[21]}$. At all temperatures, IR-HAD dried fastest, and HAD the slowest.

Infrared radiation heats the material more rapidly and uniformly without heating the surrounding air ${ }^{[25]}$. The irradiated surface evaporates much more water and the drying time can be shortened by up to half ${ }^{[26]}$ (Figure 3 ). Combining infrared radiation with hot air drying can reduce the drying time by $20 \%$ compared with using infrared radiation alone ${ }^{[27]}$.

The drying rate was affected by the moisture content of the chrysanthemum samples are shown in Figure 3, and drying rate slows as moisture content declines. In the drying process of chrysanthemum, the water loss rate of the material is controlled by the process of water transfer inside the material. As the temperature increases, the temperature inside the material also increases, and the internal water transfer is accelerated. The drying rates increased with increasing drying temperature. This phenomenon can be attributed to the effective moisture diffusivity increasing with an increase in temperature. As indicated in Figure 3 , the samples dried under IR-HAD had a higher drying rate than the samples dried by THC-HAD or HAD, at the same drying temperature, because the radiation absorbs moisture from both the surface and the interior of the sample.

Note: IR-HAD: infrared hot air-drying; THC-HAD: temperature and humidity controlled hot air-drying; HAD: hot air-drying.

Figure 3 Drying curves for chrysanthemum using different drying methods at different drying temperatures 


\subsubsection{Weibull model and $D_{\text {eff }}$}

The Weibull model was fitted to the experimental data for the drying of chrysanthemums (Table 1). All the determination coefficients $\left(R^{2}\right)$ were greater than 0.994 demonstrating the integrity of the fitting. The scale parameter $\alpha$ is related to the drying rate, and the shape parameter $\beta$ is related to the drying technique. The scale parameter $\alpha$ decreases with increasing temperature, and differs among the three drying methods. At $60^{\circ} \mathrm{C}, \alpha$ values were $105.721,112.596$, and 138.065 , for IR-HAD, THC-HAD and HAD, respectively. The $\beta$ parameter ranged from $0.942-1.286$, indicating that drying rate is fastest during the early drying phase, at all temperatures, except for the $\mathrm{HAD}$ at $50^{\circ} \mathrm{C}$ $(\beta<1)$. The Weibull model has previously been employed to describe the drying behavior of apricots ${ }^{[28]}$, rhizome dioscoreae slices $^{[29]}$, and Aloe vera gels ${ }^{[30]}$.

The effective moisture diffusivity reflects the ability of the material to dehydrate under different drying conditions. It is one of the important parameters in optimizing the design of a drying process. Because chrysanthemums are controlled by internal moisture diffusion in their drying process, Fick's second law can be used to describe the moisture $D_{\text {eff }}$ as described in Section 2.5.

The $D_{\text {eff }}$ values calculated for the chrysanthemum samples under different drying methods and drying temperatures are summarized in Table 1. The drying method and drying temperature obviously influenced $D_{e f f}$. The results show that the $D_{\text {eff }}$ increases with the increasing temperature, with the $D_{\text {eff }}$ of chrysanthemum dried by IR-HAD being the highest at the same drying temperature, followed by THC-HAD and HAD. This is also the reason for the rapid drying rate and the short drying time of IR-HAD. The $D_{\text {eff }}$ values of chrysanthemum varied from $1.38 \times 10^{-9}$ to $8.66 \times 10^{-9} \mathrm{~m}^{2} / \mathrm{s}$. This is consistent with the general law which states that the slower the effective moisture diffusivity, the longer the drying time. The moisture transfer in food varies greatly under different drying conditions, and mainly depends on the physical and chemical properties of the material ${ }^{[31]}$.

Table 1 Regression results of Weibull model, effective moisture diffusion coefficients and color of chrysanthemum under different drying techniques and temperatures

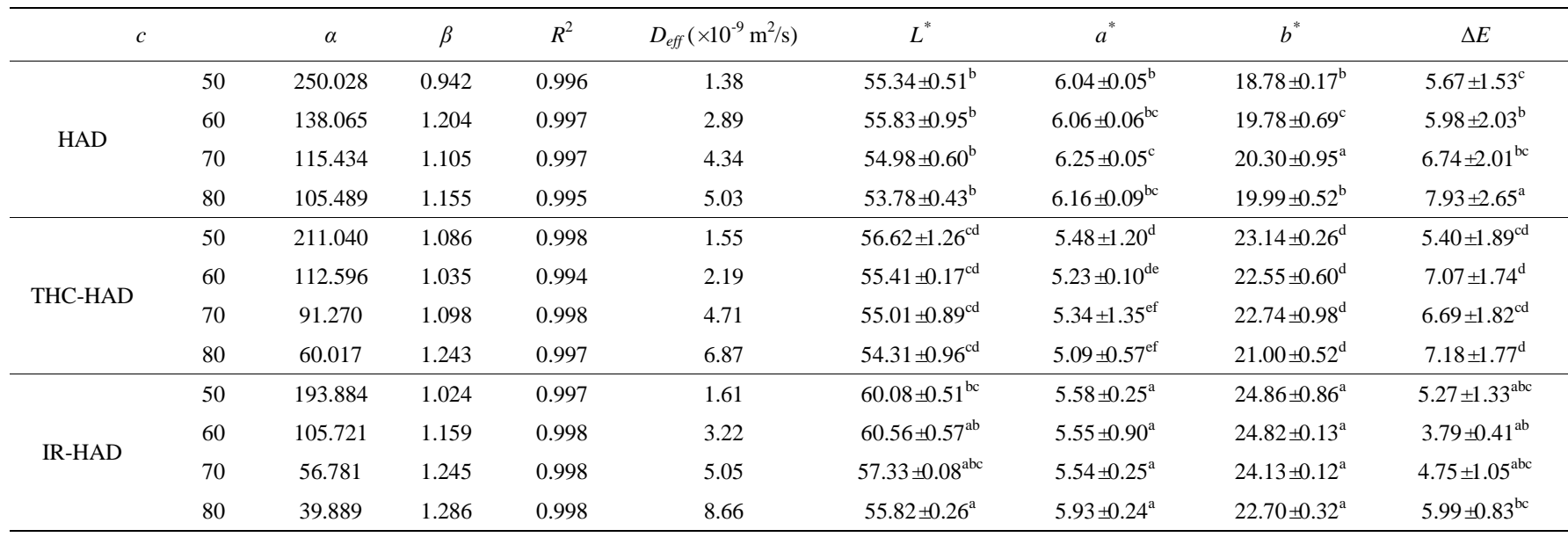

Note: IR-HAD: infrared hot air-drying; THC-HAD: temperature and humidity controlled hot air-drying; HAD: hot air-drying.

Values with the same lower case letter within each column are not significantly different $(p>0.05)$.

\subsection{Color changes}

Color is one of the most important sensory evaluation indicators for dried chrysanthemum, and a yellowish-brown color can severely restrict its acceptability and value ${ }^{[32]}$. The color parameters $L^{*}, a^{*}, b^{*}$, and $\Delta E$ of the dried samples under different drying conditions are summarized in Table 1 . It was found that with increasing drying temperature, $L^{*}$ decreased, whereas $a^{*}$ and $b^{*}$ changed much less in general. From Table 1, it was observed that samples dried at $80^{\circ} \mathrm{C}$ had significantly lower $L^{*}$ value $(p<0.05)$ than other samples, showing that high temperatures accelerated the browning reaction of chrysanthemum during drying. The $a^{*}$ value with a positive value of 60 indicates redness, and a negative value of -60 indicates greenness. Meanwhile, $b^{*}$ with a positive value of 60 indicates yellowness, whereas a negative value of -60 indicates blueness. The $a^{*}$ value of IR-HAD dried chrysanthemum was increased to $5.58,5.55,5.54$ and 5.93 at temperatures of $50^{\circ} \mathrm{C}$, $60^{\circ} \mathrm{C}, 70^{\circ} \mathrm{C}$ and $80^{\circ} \mathrm{C}$, respectively, compared to the freeze-dried samples. The total color changes, $\Delta E$ from HAD, THC-HAD, and IR-HAD exhibited clear differences. In general, the surface color of dried chrysanthemum was well preserved by IR-HAD, especially for the samples dried at $60^{\circ} \mathrm{C}$. Experiments suggested a higher color change was found in dried chrysanthemums, and the temperature significantly affected the total color changes.

\subsection{Chlorogenic acid}

Chlorogenic acid is a biologically active compound with antioxidant, antibacterial, antiviral and anti-inflammatory effects that lowers the risk of chronic and cardiovascular diseases ${ }^{[33]}$. The HPLC chromatograms of the samples are shown in Figure 4. The results indicate that the highest content of chlorogenic acid was in THC-HAD, followed by IR-HAD, and the lowest was HAD, in the three drying methods at $60^{\circ} \mathrm{C}$. This is due to the concentration of chlorogenic acid decreases with increasing of roasting times ${ }^{[34]}$. Their values are $0.112 \% \pm 0.016 \%, 0.061 \% \pm 0.003 \%$, and $0.022 \% \pm$ $0.010 \%$, respectively. The linear regression of the concentration of chlorogenic acid $(x)$ was carried out with the peak area $(y)$, and the regression equation was obtained as follows: $y=0.1449 x-0.2548$ $\left(R^{2}=0.9978\right)$.

\subsection{Water holding capacity and water binding capacity}

The water holding capacity and water binding capacity of chrysanthemum are important quality attributes, not just for the sensory aspects, but also for economic benefit. WHC and $W B C$ are usually used to characterize the water-binding characteristics of samples. The influence of drying conditions on the WHC and $W B C$ can be seen in Figure 5. The drying methods significantly affected both $W H C$ and $W B C$, whereas the drying temperature had a marginal effect on it. Chrysanthemums dried by THC-HAD had 
higher $W H C$ and $W B C$ values than those for IR-HAD and HAD. The values of $W H C$ and $W B C$ decreased with increasing temperature, which may be due to the destruction of the internal structure at high temperatures. Thermal treatments are known to destroy the cell membrane integrity of plant cells ${ }^{[35]}$. Ekaraj Paudel et al. ${ }^{[36]}$ studied the relationship between water holding capacity and cell membrane integrity loss and cell wall structure polymer state changes in hydrated mushrooms. The results showed that the loss of water holding capacity after heat treatment is closely related to the loss of cell membrane integrity.

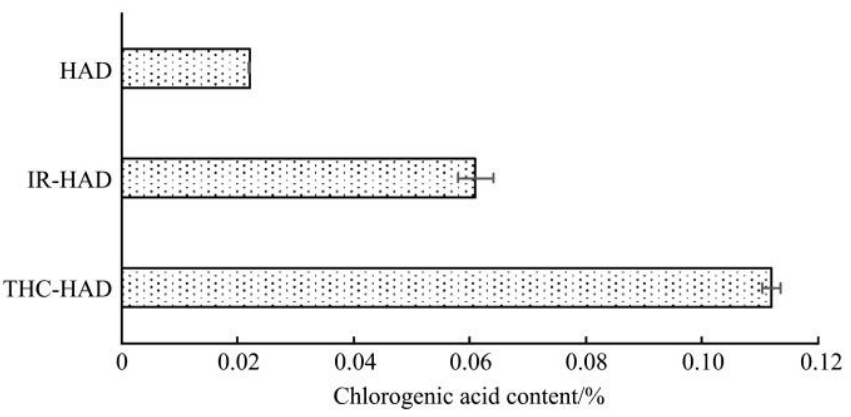

Figure 4 Chlorogenic acid content of chrysanthemum dried by different drying methods at $60^{\circ} \mathrm{C}$

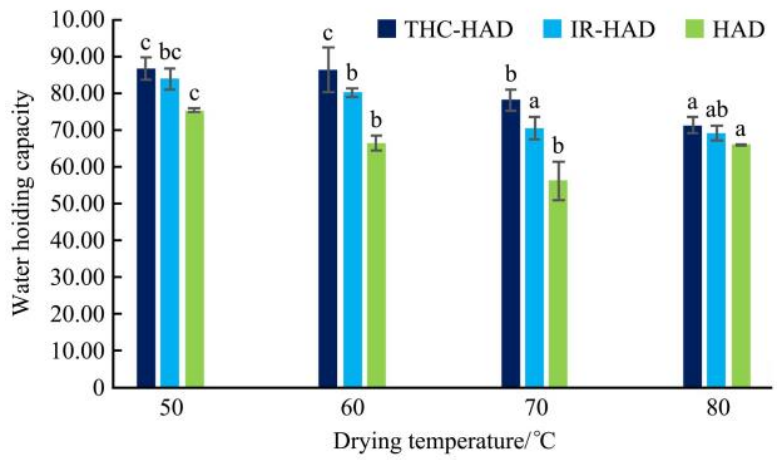

a. $W H C$

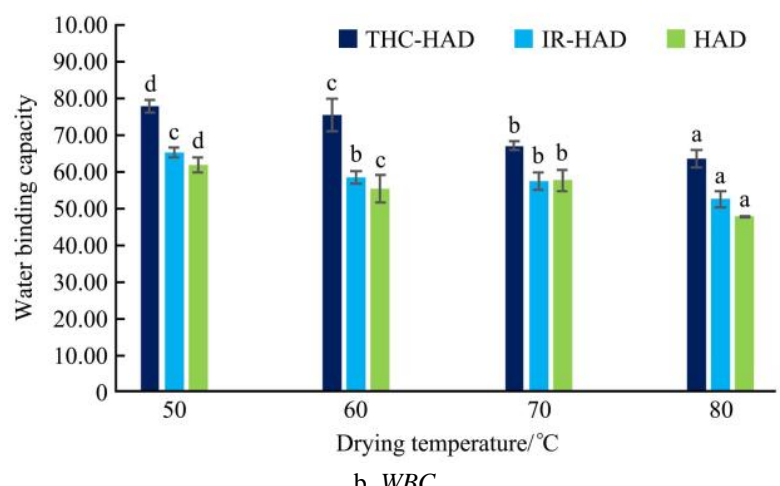

b. $W B C$

Note: IR-HAD: infrared hot air-drying; THC-HAD: temperature and humidity controlled hot air-drying; HAD: hot air-drying.

Values with the same lowercase letter at the same temperature are not significantly different $(p>0.05)$.

Figure 5 Water holding capacity $(W H C)$ and water binding capacity

$(W B C)$ of chrysanthemum dried by different drying methods

\subsection{Microstructure}

Physical or chemical changes occur during the drying process, such as shrinkage, increased porosity, poor water absorption, and damage to microstructure ${ }^{[37]}$. Figure 6 compares the microstructure of chrysanthemums dried by the three drying methods. From the micrographs, it can be observed that the powder of the chrysanthemum samples had distinct bulges and a smooth surface structure. Evident powder structure changes were observed among the different dried samples. It was found that in the HAD samples the surface bulges and micro-cracks increased, and there were visibly adsorbed small particles (Figure 6a). While the microstructure resulting from THC-HAD (Figure 6b) and IR-HAD (Figure 6c) had fewer surface bulges and micro-cracks, and IR-HAD resulted in the smoothest fragments. The very rough fragments observed after grinding HAD flowers is because HAD requires a high energy supply, which can lead to severe shrinkage, reduced bulk density and rehydration capacity, especially when high temperatures are used during the drying process. With the novel thermal IR-HAD method, simultaneously combined with convective hot-air, overcomes most of the shortcomings associated with convective $\mathrm{HAD}^{[38]}$.

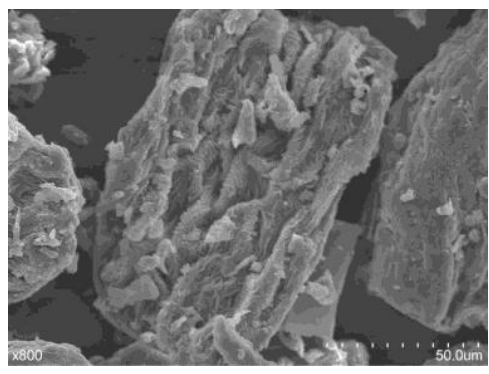

a. HAD

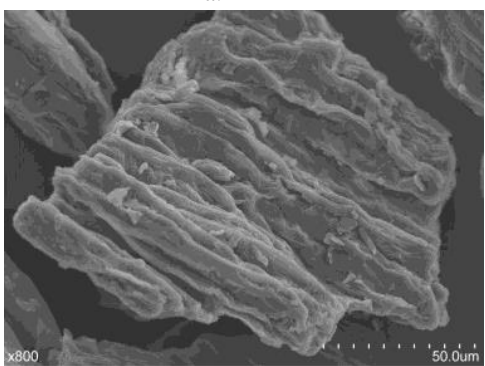

b. THC-HAD

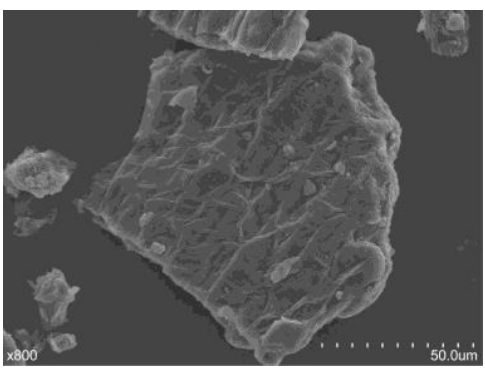

c. IR-HAD

Figure 6 Scanning electron micrograph $(800 \times$ magnifications $)$ of chrysanthemum under different drying methods at $60^{\circ} \mathrm{C}$

\section{Conclusions}

The effects of three different drying methods (HAD, THC-HAD, and IR-HAD) on the drying kinetics, physicochemical properties, chlorogenic acid content, and microstructure of chrysanthemum were investigated. The IR-HAD method was more effective than THC-HAD and HAD in decreasing the drying time. The increase of the temperature followed by high effective moisture diffusivity improved the drying rate and decreased the total drying time. In particular, the studies indicate that IR-HAD could ensure the quality of chrysanthemum at $60^{\circ} \mathrm{C}$, as it resulted in a better surface color. On the other hand, THC-HAD resulted in a higher $W H C$ and $W B C$, and had a higher content of chlorogenic acid when compared to IR-HAD and HAD. In addition, the $P O D$ activity decreased with increasing blanching times. These findings provide a theoretical and technical basis for the drying of chrysanthemums. 


\section{Acknowledgement}

This work was supported by China Agriculture Research System (CARS-21). We would like to acknowledge Bioenergy Environmental Science and Technology Research Lab, College of Engineering, China Agricultural University, for the use of the DIONEX high performance liquid chromatography. Furthermore, the authors would like to thank PhD students Yongkang Xie and Hui Sun for helping in this study.

\section{[References]}

[1] Wang Y, Li X, Chen X, Li B, Mao X, Miao J, et al. Effects of hot air and microwave-assisted drying on drying kinetics, physicochemical properties, and energy consumption of chrysanthemum. Chemical Engineering and Processing - Process Intensification, 2018; 129: 84-94.

[2] Lu D, Zhiqiang H, Di L, Pengfang Z, Shengjin L, Na L, et al. Transcriptome analysis of chrysanthemum in responses to white rust. Sci Hortic-Amsterdam, 2018; 233: 421-430.

[3] Pharmacopoeia of the People's Republic of China. GB/T 23183-2009. Beijing: Chemical Industry Press, 2015. (in Chinese)

[4] Liu F, Ong E S, Li S F Y. A green and effective approach for characterisation and quality control of Chrysanthemum by pressurized hot water extraction in combination with HPLC with UV absorbance detection. Food Chem, 2013; 141(3): 1807-1813.

[5] Nam S, Ko J, Jun W, Wee Y, Walsh M K, Yang K, et al. Enzymatic synthesis of chlorogenic acid glucoside using dextransucrase and its physical and functional properties. Enzyme Microb Tech, 2017; 107: 15-21.

[6] Yuan J, Hao L, Wu G, Wang S, Duan J, Xie G, et al. Effects of drying methods on the phytochemicals contents and antioxidant properties of chrysanthemum flower heads harvested at two developmental stages. J Funct Foods, 2015; 19: 786-795.

[7] He J, Chen F, Chen S, Lv G, Deng Y, Fang W, et al. Chrysanthemum leaf epidermal surface morphology and antioxidant and defense enzyme activity in response to aphid infestation. J Plant Physiol, 2011; 168(7): 687-693.

[8] Bai J, Sun D, Xiao H, Mujumdar A S, Gao Z. Novel high-humidity hot air impingement blanching (HHAIB) pretreatment enhances drying kinetics and color attributes of seedless grapes. Innov Food Sci Emerg, 2013; 20: 230-237.

[9] Yang X, Zhang Q, Wang J, Deng L, Kan Z. Innovative superheated steam impingement blanching (SSIB) enhances drying rate and quality attributes of line pepper. Information Processing in Agriculture, 2017; 4(4): 283-290

[10] Wang J, Mujumdar AS, Deng L, Gao Z, Xiao H, Raghavan G S V. High-humidity hot air impingement blanching alters texture, cell-wall polysaccharides, water status and distribution of seedless grape. Carbohyd Polym, 2018; 194: 9-17.

[11] Deng L, Mujumdar A S, Yang X, Wang J, Zhang Q, Zheng Z, et al. High humidity hot air impingement blanching (HHAIB) enhances drying rate and softens texture of apricot via cell wall pectin polysaccharides degradation and ultrastructure modification. Food Chem, 2018; 261: 292-300.

[12] Xiao H, Bai J, Xie L, Sun D, Gao Z. Thin-layer air impingement drying enhances drying rate of American ginseng (Panax quinquefolium L.) slices with quality attributes considered. Food Bioprod Process, 2015; 94: 581-591.

[13] Zhu Y, Pu B, Xie G, Tian M, Xu F, Qin M. Dynamic changes of flavonoids contents in the different parts of rhizome of belamcanda chinensis during the thermal drying process. Molecules, 2014; 19(7):10440-10454.

[14] Sehrawat R, Nema PK, Kaur B P. Quality evaluation and drying characteristics of mango cubes dried using low-pressure superheated steam, vacuum and hot air drying methods. LWT - Food Science and Technology, 2018; 92: 548-555.

[15] Ding C, Khir R, Pan Z, Wood D F, Venkitasamy C, Tu K, et al. Influence of infrared drying on storage characteristics of brown rice. Food Chem, 2018; 264: 149-156.

[16] Younis M, Abdelkarim D, Zein El-Abdein A. Kinetics and mathematical modeling of infrared thin-layer drying of garlic slices. Saudi J Biol Sci, 2018; 25(2): 332-338
[17] Ju H, Law C, Fang X, Xiao H, Liu Y, Gao Z. Drying kinetics and evolution of the sample's core temperature and moisture distribution of yam slices (Dioscorea alata L.) during convective hot-air drying. Dry Technol, 2016; 34(11): 1297-1306.

[18] National Standards of People's Republic of China. Determination of moisture in food, GB/T 5009.3-2016; Beijing: Standards Press of China, 2016. (in Chinese)

[19] Bai J, Gao Z, Xiao H, Wang X, Zhang Q. Polyphenol oxidase inactivation and vitamin $\mathrm{C}$ degradation kinetics of Fuji apple quarters by high humidity air impingement blanching. International Journal of Food Science \& Technology, 2013; 48(6): 1135-1141.

[20] Xiao H, Bai J, Sun D, Gao Z. The application of superheated steam impingement blanching (SSIB) in agricultural products processing-A review. J Food Eng, 2014; 132: 39-47.

[21] Deng L, Yang X, Mujumdar A S, Zhao J, Wang D, Zhang Q, et al. Red pepper (Capsicum annuum L.) drying: Effects of different drying methods on drying kinetics, physicochemical properties, antioxidant capacity, and microstructure. Dry Technol, 2018; 36(8): 893.

[22] Shi X, Chu J, Zhang Y, Liu C, Yao X. Nutritional and active ingredients of medicinal chrysanthemum flower heads affected by different drying methods. Ind Crop Prod, 2017; 104: 45-51.

[23] Wang J, Yang X, Mujumdar A S, Wang D, Zhao J, Fang X, et al. Effects of various blanching methods on weight loss, enzymes inactivation, phytochemical contents, antioxidant capacity, ultrastructure and drying kinetics of red bell pepper (Capsicum annuиm L.). LWT - Food Science and Technology, 2017; 77: 337-347.

[24] Xiao H, Law C, Sun D, Gao Z. Color change kinetics of American ginseng (Panax quinquefolium) slices during air impingement drying. Dry Technol, 2014; 32(4): 418-427.

[25] Adak N, Heybeli N, Ertekin C. Infrared drying of strawberry. Food Chem, 2017; 219: 109-116.

[26] Nowak D, Lewicki P P. Infrared drying of apple slices. Innov Food Sci Emerg, 2004; 5(3): 353-360.

[27] Sun J, Hu X, Zhao G, Wu J, Wang Z, Chen F, et al. Characteristics of thin-layer infrared drying of apple pomace with and without hot air pre-drying. Food Sci Technol Int, 2016; 13(2): 91-97.

[28] Dai J, Rao J, Wang D, Xie L, Xiao H, Liu Y, et al. Process-based drying temperature and humidity integration control enhances drying kinetics of apricot halves. Dry Technol, 2015; 33(3): 365-376.

[29] Xie Y, Gao Z, Liu Y, Xiao H. Pulsed vacuum drying of rhizoma dioscoreae slices. LWT-Food Science and Technology, 2017; 80: 237-249.

[30] Miranda M, Vega-Gálvez A, García P, Di Scala K, Shi J, Xue S, et al. Effect of temperature on structural properties of Aloe vera (Aloe barbadensis Miller) gel and Weibull distribution for modelling drying process. Food Bioprod Process, 2010; 88(2-3): 138-144.

[31] Anabel F, Celia R, Germán M, Rosa R. Determination of effective moisture diffusivity and thermodynamic properties variation of regional wastes under different atmospheres. Case Studies in Thermal Engineering, 2018; 12: 248-257.

[32] Wang J, Law C, Nema P K, Zhao J, Liu Z, Deng L, et al. Pulsed vacuum drying enhances drying kinetics and quality of lemon slices. J Food Eng, 2018; 224: 129-138.

[33] Meinhart A D, Damin F M, Caldeirão L, da Silveira TFF, Filho J T, Godoy $\mathrm{H}$ T. Chlorogenic acid isomer contents in 100 plants commercialized in Brazil. Food Res Int, 2017; 99: 522-530.

[34] Moon J, Yoo H S, Shibamoto T. Role of roasting conditions in the level of chlorogenic acid content in coffee beans: Correlation with coffee acidity. J AGR Food Chem, 2009; 57(12): 5365-5369.

[35] Gonzalez M E, Barrett D M, McCarthy M J, Vergeldt F J, Gerkema E, Matser A M, et al. 1H-NMR study of the impact of high pressure and thermal processing on cell membrane integrity of Onions. J Food Sci, 2010; 75(7): E417-425.

[36] Paudel E, Boom R M, van Haaren E, Siccama J, van der Sman R G M Effects of cellular structure and cell wall components on water holding capacity of mushrooms. J Food Eng, 2016; 187: 106-113.

[37] Witrowa-Rajchert $\mathrm{D}, \mathrm{Rz} \mathrm{Ca}$ M. Effect of drying method on the microstructure and physical properties of dried apples. Dry Technol, 2009; 27(7-8): 903-909.

[38] Onwude D I, Hashim N, Abdan K, Janius R, Chen G, Kumar C. Modelling of coupled heat and mass transfer for combined infrared and hot-air drying of sweet potato. J Food Eng, 2018; 228: 12-24. 